\title{
Utilização de uma abordagem dietética no controle e redução dos riscos cardiovasculares em pacientes com hipertensão arterial sistêmica
}

\author{
Use of a dietary approach to control and reduce cardiovascular risks in patients with \\ systemic arterial hypertension
}

Uso de un enfoque dietético para controlar y reducir los riegos cardiovasculares em pacientes con hipertesión arterial sistémica

Niccoly Kolle Pereira ${ }^{1 *}$, Breno Carvalho Tambuque de Oliveira ${ }^{2}$, Bruno Carvalho Tambuque de Oliveira $^{2}$, Clelio Mendes da Silva Filho ${ }^{3}$, Douglas Almeida Costa ${ }^{4}$, Igor Francisco Matoso da Fonseca ${ }^{4}$, Laise Soares Costa ${ }^{4}$, Tales de Sousa Coutinho Ferreira Pires ${ }^{3}$, Luciano Coelho de Souza ${ }^{5}$.

\section{RESUMO}

Objetivo: Estudar a eficácia clínica da Dietary Approaches to Stop Hypertension (dieta DASH) no controle da Hipertensão Arterial Sistêmica (HAS) e, por conseguinte, redução dos riscos de doenças cardiovasculares. Revisão bibliográfica: A prevalência da HAS aumenta com a idade. Tendo em vista o crescimento da população idosa no país, essa condição clínica se apresenta como um desafio para o sistema público de saúde. Existem, de maneira geral, duas abordagens principais para o tratamento da hipertensão arterial: a farmacológica com a utilização dos anti-hipertensivos e a não farmacológica como a melhoria dos hábitos de vida. A dieta DASH faz parte do grupo dessas intervenções não medicamentosas e consiste em uma maior ingestão de frutas, verduras, produtos com baixo teor de sódio, colesterol e ácidos graxos saturados. Esse padrão alimentar possui benefícios reconhecidos na redução da pressão arterial sistêmica e riscos cardiovasculares, uma vez que o consumo de sal e colesterol é menor, contribuindo, assim, para a prevenção de acidentes coronarianos e vasculares cerebrais. Considerações finais: Por esses motivos, a dieta DASH tem sido apontada por diretrizes nacionais e internacionais como medida terapêutica para o controle da hipertensão arterial sistêmica.

Palavras-chave: Hipertensão arterial sistêmica, Dieta DASH, Riscos cardiovasculares.

\begin{abstract}
Objective: To study the clinical effectiveness of the Dietary Approaches to Stop Hypertension (DASH diet) in the control of Systemic Arterial Hypertension (SAH) and, consequently, reducing the risk of cardiovascular diseases. Literature review: The prevalence of SAH increases with age. In view of the growth of the elderly population in the country, this clinical condition presents itself as a challenge for the public health system. There are, in general, two main approaches for the treatment of arterial hypertension: the pharmacological one, with the use of antihypertensive drugs, and the non-pharmacological one, such as improving lifestyle habits. The DASH diet is part of the group of these non-drug interventions and consists of a greater intake of fruits, vegetables, products with low sodium content, cholesterol and saturated fatty acids. This dietary pattern

1 Universidade Cidade de São Paulo (UNICID), São Paulo - SP. *E-mail: niccolykolle@gmail.com

2 União Metropolitana de Educação e Cultura (UNIME), Lauro de Freitas - BA.

${ }^{3}$ Universidade de Vassouras (UV), Vassouras - RJ.

${ }^{4}$ Faculdade Dinâmica do Vale do Piranga (FADIP), Ponte Nova - MG.

${ }^{5}$ Universidade Federal de Minas Gerais (UFMG), Belo Horizonte - MG.
\end{abstract}

SUBMETIDO EM: 6/2021

ACEITO EM: 7/2021

PUBLICADO EM: 8/2021 
has recognized benefits in reducing systemic blood pressure and cardiovascular risks, since the consumption of salt and cholesterol is lower, thus contributing to the prevention of coronary and cerebrovascular accidents. Final considerations: For these reasons, the DASH diet has been identified by national and international guidelines as a therapeutic measure for the control of systemic arterial hypertension.

Key words: Systemic arterial hypertension, DASH diet, Cardiovascular risks.

\section{RESUMEN}

Objetivo: Estudiar la efectividad clínica de los Enfoques Dietéticos para Detener la Hipertensión (dieta DASH) en el control de la Hipertensión Arterial Sistémica (HSA) y, en consecuencia, la reducción del riesgo de enfermedades cardiovasculares. Revisión de la literatura: la prevalencia de HSA aumenta con la edad. Ante el crecimiento de la población anciana en el país, esta condición clínica se presenta como un desafío para el sistema de salud pública. Existen, en general, dos enfoques principales para el tratamiento de la hipertensión arterial: el farmacológico, con el uso de fármacos antihipertensivos, y el no farmacológico, como la mejora de los hábitos de vida. La dieta DASH forma parte del grupo de estas intervenciones no farmacológicas y consiste en una mayor ingesta de frutas, verduras, productos con bajo contenido en sodio, colesterol y ácidos grasos saturados. Este patrón dietético tiene reconocidos beneficios en la reducción de la presión arterial sistémica y los riesgos cardiovasculares, ya que el consumo de sal y colesterol es menor, contribuyendo así a la prevención de accidentes coronarios y cerebrovasculares. Consideraciones finales: Por estas razones, la dieta DASH ha sido identificada por guías nacionales e internacionales como una medida terapéutica para el control de la hipertensión arterial sistémica.

Palabras clave: Hipertensión arterial sistémica, Dieta tablero, Riesgos cardiovasculares.

\section{INTRODUÇÃO}

Nas últimas décadas, as doenças cardiovasculares estão entre as que mais crescem ao redor do mundo. De acordo com dados da Pesquisa Nacional da Saúde (PNS), estima-se que o Brasil, no ano de 2025, apresentará a sexta maior população na faixa etária idosa, tendo em média 30 milhões de pessoas. Como consequência, é esperado uma multiplicação dos casos de hipertensão, diabetes e outros problemas de saúde, assim como aumento dos gastos e sobrecarga no sistema público de saúde com tais doenças crônicas (MALACHIAS MVB, et al., 2016; SOCIEDADE BRASILEIRA DE CARDIOLOGIA (SBC), 2016).

A prevalência de Hipertensão Arterial Sistêmica (HAS) aumenta com a idade (mais de dois terços das pessoas com mais de 65 anos são hipertensas), com o sedentarismo e, consequentemente, com a presença de obesidade. É uma condição clínica multifatorial identificada por níveis elevados e sustentados da pressão arterial, destacando-se atualmente como uma doença cardiovascular com alto impacto na morbimortalidade. Comumente, está associada com alterações metabólicas, funcionais e/ou estruturais dos órgãos alvos (coração, encéfalo, rins e vasos sanguíneos), corroborando ao desenvolvimento de um maior risco de doenças potencialmente fatais e não fatais, tais como: insuficiência cardíaca, infarto agudo do miocárdio, acidente vascular cerebral, insuficiência renal crônica, aneurisma, dissecção de aorta e retinopatia hipertensiva (MALTA DC, et al., 2017).

O tratamento da HAS consiste em métodos farmacológicos e não farmacológicos. Os farmacológicos estão divididos em diferentes classes de anti-hipertensivos e são indicados de forma individualizada a cada paciente de acordo com sua pressão arterial, presença de doença cardiovascular ou fatores de risco; já os não farmacológicos são aqueles que exigem determinação do paciente em busca de uma melhora na qualidade de vida e redução dos riscos cardiovasculares, como atividade física regular, cessação do tabagismo, redução do peso ponderal, redução do consumo de sódio e inclusão de dietas, como a Dietary Approach to Stop Hypertension (DASH) que tem sido cada vez mais abordada entre os profissionais da saúde (GEWEHR DM, et al., 2018; SBC, 2016). 
A dieta DASH vem sendo recomendada em diretrizes nacionais e internacionais como medida terapêutica, ao preconizar o consumo de frutas, verduras, produtos lácteos com baixo teor de gordura, cereais integrais, peixe, aves, ao passo que incentiva um menor consumo de carnes vermelhas, doces e açúcares. Seus benefícios decorrem de quantidades significativas de macro/micronutrientes de cálcio, potássio, magnésio e ácidos graxos insaturados em sua composição, e o uso limitado de sódio, colesterol e ácidos graxos saturados. Posto isso, seu propósito principal é a inserção de nutrientes com potencial efeito hipotensor, contribuindo para redução dos níveis pressóricos (BRICARELLO LP, et al., 2020).

Diante do exposto, o presente artigo tem como objetivo ratificar, por meio de uma revisão narrativa, a eficácia clínica da dieta DASH na prevenção e controle da HAS.

\section{REVISÃO BIBLIOGRÁFICA}

\section{Definição e epidemiologia}

De acordo com a 7a Diretriz Brasileira de Hipertensão Arterial, redigida pela SBC, a HAS trata-se de uma condição clínica que se caracteriza por elevações dos níveis pressóricos sustentadas que são maiores ou iguais a $140 \mathrm{mmHg}$ na sístole e maior ou igual a $90 \mathrm{mmHg}$ na diástole. Tal enfermidade se associa com vasta frequência de distúrbios metabólicos, alterações estruturais e funcionais de órgão alvo. No Brasil, cerca de $32,5 \%$ dos indivíduos adultos e $60 \%$ dos idosos são portadores de HAS. Ademais, essa doença está direta ou indiretamente contribuindo para aproximadamente $50 \%$ das mortes advindas de doenças cardiovasculares (DCV), que é atualmente a principal causa de morte no país, ficando evidente a necessidade de controle e redução da HAS (SBC, 2016; LONGO MAT, et al., 2011).

A HAS é agravada quando em conjunto a certos fatores de risco, como Diabetes Mellitus (DM), dislipidemia, intolerância à glicose e obesidade abdominal. Há também ligação de forma independente a eventos como Infarto Agudo do Miocárdio (IAM), Acidente Vascular Cerebral (AVC), Insuficiência Cardíaca (IC), Doença Arterial Periférica (DAP), Doença Renal Crônica (DRC) e Morte Súbita (SBC, 2016).

\section{Fisiopatologia da Hipertensão Arterial Sistêmica (HAS)}

A pressão arterial (PA) é determinada pelo produto do Débito Cardíaco (DC) com a Resistência Vascular Periférica (RVP). O DC e RVP são regulados por mecanismos neurais, renais, humorais, endoteliais e locais de controle das funções cardiovasculares e renais (LONGO MAT, et al., 2011; MELGAÇO NPR, et al., 2021).

Referente aos mecanismos neurais, o Sistema Nervoso Simpático (SNS) promove o aumento da RVP, da contratilidade e da Frequência Cardíaca (FC), mantendo assim o controle em curto prazo da PA (PASSOS VMA, et al., 2006).

Relativo aos mecanismos renais, a influência do complexo renal na PA se dá pelo controle do equilíbrio hidrossalino, da autorregulação renal, dos ajustes da excreção de sódio e da ativação do Sistema ReninaAngiotensina (SRA). Infere-se que a HAS advinda dos mecanismos renais acontece devido a uma redução numérica de néfrons ou da superfície de filtração por glomérulo, fazendo com que a excreção renal de sódio seja reduzida e, consequentemente, gere aumento da PA (PASSOS VMA, et al., 2006; MALTA DC, et al., 2017).

Relacionado aos mecanismos humorais, o SRA também é fundamental no equilíbrio hidroeletrolítico e está envolvido no controle fisiológico da PA. A sua atividade persistentemente elevada está associada com o aumento da pressão arterial. Isso acontece devido à angiotensina II, principal componente do SRA, que é responsável por efeitos pró-hipertensivos quando em contato com seu receptor do tipo 1 (AT1), tais como: vasoconstrição sistêmica e renal, hipertrofia e hiperplasia da musculatura lisa vascular, elevação do débito cardíaco, retenção renal de sódio e água, proteinúria, estímulo à secreção de aldosterona pelas adrenais, disfunção endotelial, hipertensão glomerular, hipertrofia cardíaca e resistência à insulina. Além disso, a angiotensina II age estimulando a resposta inflamatória e a fibrose tecidual, que contribuem para as lesões de órgãos alvo na HAS (PASSOS VMA, et al., 2006). 
Pode-se afirmar nos mecanismos genéticos, a existência de vários polimorfismos funcionais em genes do SRA ligados à HAS. Dentre eles, é o polimorfismo de inserção e deleção (I/D) do gene da ECA. Os resultados de estudos realizados acerca de polimorfismos no Sistema Renina-Angiotensina-Aldosterona (SRAA) demonstram que os participantes com 0 alelo $D$ do gene da ECA mostram formação mais elevada de angiotensina II no coração, nos vasos sanguíneos e no tecido renal. Leves diferenças genéticas são importantes quando se considera sua interação com os fatores ambientais e o papel que poderá desenvolver na regulação da PA e na resposta ao tratamento (PASSOS VMA, et al., 2006).

\section{Fatores de risco modificáveis e não modificáveis para desenvolver HAS}

\section{Fatores modificáveis}

A OMS recomenda que o consumo máximo de sal seja de até $2 \mathrm{~g} / \mathrm{dia}$. No Brasil, segundo dados da Pesquisa de Orçamentos Familiares, se excede consideravelmente, sendo a disponibilidade domiciliar de $4,7 \mathrm{~g}$ de sódio/pessoa/dia. Estudos inferem que uma dieta em que os indivíduos fazem uma menor ingestão de sódio e maior em potássio, apresentam PA mais baixa que em casos contrários. Contudo, vale ressaltar que a influência dietética sobre a PA é relativa à sensibilidade do indivíduo ao sódio. De todo modo, a redução de ingestão de sal na dieta pode provocar diminuição da PA sistólica suficiente para diminuir o risco de lesões em órgãos alvo (JURASCHEK SP, et al., 2017; SBC, 2016; WESCHENFELDER MD e GUE MJ, 2011).

De acordo com a ingestão de bebidas alcoólicas, mulheres com o consumo alcoólico de 30-40 g/dia apresentaram maior risco de HAS. Em homens, o risco de HAS tornou-se aumentado a partir de $31 \mathrm{~g}$ de álcool/dia (SBC, 2016).

Além disso, a obesidade merece um destaque dentre os principais fatores de risco da HAS, visto que leva ao aumento da produção de insulina e de leptina circulantes na corrente sanguínea. Deste modo, tem-se a hiperinsulinemia que propicia possíveis alterações estruturais e funcionais da musculatura lisa vascular e disfunção endotelial que podem causar HAS. O Ministério da Saúde relata que a prevalência entre homens e mulheres portadores de HAS e obesidade $\left(\mathrm{IMC}>30 \mathrm{~kg} / \mathrm{m}^{2}\right)$ seja de respectivamente $70 \%$ e $60 \%$ (KUCHARSKA A, et al., 2018; SBC, 2016).

\section{Fatores não modificáveis}

O avançar da idade pode contribuir para o aparecimento de fatores de risco não modificáveis e complicações a curto e longo prazo para as DCV, pois a HAS tem ligação direta e linear com o envelhecimento. No Brasil, $68 \%$ da população idosa (> 60 anos) possui HAS (SBC, 2016; JURASCHEK SP, et al., 2017).

O estudo Corações do Brasil revelou a seguinte distribuição de HAS por raças: $11,1 \%$ na população indígena; $10 \%$ na amarela; $26,3 \%$ na parda/mulata; $29,4 \%$ na branca e 34,8\% na negra. Já o estudo ELSABrasil mostrou prevalências de 30,3\% em brancos, 38,2\% em pardos e 49,3\% em negros (SBC, 2016; JURASCHEK SP, et al., 2017).

\section{Tratamento farmacológico}

Ao optar por um esquema farmacológico terapêutico, a monoterapia ou a combinação de fármacos pode ser utilizada. A monoterapia pode ser usada em pacientes com HAS estágio $1 \mathrm{com}$ baixo risco cardiovascular ou pré-hipertensos de alto risco cardiovascular. Não se deseja uma redução muito acentuada da pressão arterial nesses pacientes (SBC, 2016; MALTA DC, et al., 2017).

Os principais anti-hipertensivos utilizados são: diuréticos tiazídicos, bloqueadores do canal de cálcio (BCC), inibidores da enzima conversora de angiotensina (IECA) e os bloqueadores dos receptores AT1 de angiotensina II (BRA). Os betabloqueadores podem ser utilizados em situações específicas. Independentemente do estágio e do risco cardiovascular, a combinação de fármacos é a estratégia preferencial para a maioria dos hipertensos. Inicialmente, faz-se uma associação dupla utilizando medicamentos com funcionalidades diferentes, exceto o uso de diuréticos poupadores de potássio e os tiazídicos. Ressalta-se que combinações triplas podem ser feitas (SBC, 2016; MALTA DC, et al., 2017). 
Dentro das principais classes de anti-hipertensivos, encontram-se os diuréticos. O efeito anti-hipertensivo dos diuréticos não guarda relação com a dose ingerida, no entanto, os efeitos colaterais estão intimamente ligados. Além disso, deve-se dar preferência à escolha dos diuréticos tiazídicos, como a hidroclorotiazida, em doses baixas, para redução de efeitos colaterais. Os diuréticos poupadores de potássio, como a espironolactona, geralmente é utilizado com diuréticos tiazídicos e diuréticos de alça. Porém, a espironolactona é utilizada como quarto medicamento ou para HAS refratária ou persistente. Nesse contexto, os principais efeitos colaterais dos diuréticos são câimbras, fraqueza, disfunção erétil e hipovolemia (SBC, 2016).

Os BCC diminuem a quantidade de cálcio no interior das células musculares lisas, dificultando o processo de contração muscular, que, por sua vez, diminui a resistência vascular periférica por vasodilatação. Os BCC podem ser classificados de acordo com suas ações em: di-hidropiridínicos e não dihidropiridínicos. Os não di-hidropiridínicos possuem influência na funcionalidade cardíaca, sendo responsáveis por inibição do nódulo átrio ventricular, o que gera efeitos antiarrítmicos e redução da frequência cardíaca, bem como redução da função sistólica. Assim, seu uso em indivíduos usuários de betabloqueadores deve ser cauteloso. Já os dihidropiridínicos, apresentam maior função redutora da pressão arterial, com pouca interferência na funcionalidade cardíaca, sendo, portanto, mais utilizado no tratamento anti-hipertensivo, embora possa causar taquicardia reflexa inicial (MACHADO LA et al., 2021).

Além da diminuição da pressão arterial, os IECA estão associados a uma diminuição do remodelamento cardiovascular pós IAM e retardo no declínio da função renal em pacientes nefropatas. Normalmente, os IECA são bem tolerados, tendo a tosse seca como sintoma principal, presente em cerca de 5 a $20 \%$ dos pacientes. Já os BRA, especialmente em pacientes de alto risco cardiovascular, oferecem uma redução da morbidade e da mortalidade cardiovascular e renal. Efeitos adversos normalmente não são observados na utilização dos BRA (SBC, 2016).

\section{Tratamento não farmacológico}

\section{Dieta DASH}

Algumas formas de se alimentar entraram em pauta a fim de reduzir a quantidade de mortes pela hipertensão arterial que acomete milhões de pessoas no mundo, como a dieta do mediterrâneo e a dieta DASH. Apesar de ser uma dieta saudável, que reduz a incidência de problemas cardiovasculares e que se assemelha em alguns aspectos à DASH, (rica em hortaliças, frutas, cereais, pobre em carne vermelha), a dieta do mediterrâneo está associada a resultados modestos em relação a diminuição da pressão arterial (CHALLA HJ, et al., 2020; FILIPPOU CD, et al., 2020; MARIS SA, et al., 2019).

A dieta DASH é considerada, atualmente, a principal intervenção não farmacológica dietética no controle da HAS. Esse padrão alimentar se baseia em um maior consumo diário de frutas, hortaliças, laticínios com baixo teor de gordura e cereais integrais, assim como uma redução acentuada no consumo de alimentos ricos em lipídios e açúcares, resultando em menor ingesta de oleaginosas, doces, bebidas açucaradas e carne vermelha. É importante salientar que nenhum componente da dieta DASH isolado possui o efeito redutor da pressão arterial. Na verdade, é o padrão alimentar diário, o responsável pelo efeito hipotensor (FILIPPOU CD, et al., 2020; BARROSO WKS, et al., 2021).

Um estudo que reuniu 412 indivíduos que foram designados a ingerir uma dieta típica americana ou a dieta DASH, mostrou que houve uma redução significativa dos níveis pressóricos no grupo que se alimentou da dieta DASH. Além disso, foi verificado que a ingesta da DASH associada a uma redução do consumo de sódio, resultou em diminuição da Pressão Arterial Sistólica (PAS) em $11,5 \mathrm{mmHg}$ em indivíduos hipertensos, bem como uma redução de $7,1 \mathrm{mmHg}$ em indivíduos normotensos. Outra pesquisa constatou que a combinação da DASH com a diminuição do sódio era a mais eficaz no controle da pressão arterial do que a utilização de uma estratégia isoladamente. Assim, é importante mencionar sobre a influência da dieta DASH combinada à redução da ingesta de sódio na população negra, visto que os negros possuem uma tendência maior à HAS (BARROSO WKS, et al., 2021; MARIS SA, et al., 2019). 
Outro estudo clínico randomizado multicêntrico foi realizado a fim de analisar dois tipos de padrões alimentares: uma dieta tipicamente americana e a dieta DASH. Os indivíduos que participaram da pesquisa não eram portadores de HAS ou possuíam HAS leve. Foram separados em três grupos: Dieta tipicamente americana, dieta rica em frutas e vegetais e, por fim, uma dieta combinada (rica em frutas, vegetais, reduzida em gordura. Os três grupos consumiram, aproximadamente, a mesma quantidade de sal ( $3 \mathrm{~g} / \mathrm{dia})$. Em relação ao controle (dieta tipicamente americana), ambos os grupos experimentaram redução dos valores pressóricos, em especial o grupo da dieta combinada, que obteve uma média de redução da pressão arterial de 11,4 mmHg na PAS e de 5,5 mmHg na pressão arterial diastólica (PAD) (PIPER AV, et al., 2012).

Em um estudo patrocinado pelo National Heart, Lung and Blood Institute nos Estados Unidos, que visava observar a influência dietética na redução da PA, reuniram-se 459 adultos com idade média de 44 anos, onde 133 eram hipertensos e 326 não eram hipertensos. Os indivíduos receberam três tipos de dietas: dieta tipicamente americana, dieta tipicamente americana com maior quantidade de frutas e verduras e a mesma dieta citada, porém, com adição de laticínios desnatados e carnes magras (DASH) por 8 semanas. Os resultados mostraram redução dos níveis pressóricos nos dois grupos que consumiram maior quantidade de frutas e vegetais. No entanto, o grupo da dieta DASH obteve a maior redução da pressão em apenas 2 semanas de seguimento do estudo (MACHADO LA, et al. 2021).

Um estudo multicêntrico randomizado foi feito com 810 pessoas (HAS leve e não hipertensos), para verificar se há redução de valores pressóricos com a inserção da dieta DASH. Esses indivíduos foram randomizados em três grupos: o primeiro grupo recebeu orientações padrões, como perda de peso, diminuição da ingesta de bebidas alcoólicas e atividade física $(n=268)$. Já o segundo grupo também adotou orientações padrões, mas associada à dieta DASH como padrão alimentar $(n=269)$ e o terceiro grupo recebeu apenas informação nutricional em um único momento no começo da pesquisa $(n=273)$. Ao fim da pesquisa, seis meses após, o grupo de orientações padrões sem dieta DASH obteve redução de $10,5 \mathrm{mmHg}$ na PAS e $5,5 \mathrm{mmHg}$ na PAD. Já o grupo que recebeu orientações padrões com a dieta DASH, experimentou redução de 11,1 mmHg na PAS e 6,4 mmHg na PAD (PIPER AV, et al., 2012).

Uma pesquisa selecionou pessoas com pré-hipertensão e HAS grau I. Ao aplicar a dieta DASH com restrição parcial do sódio, foi verificada a redução dos valores pressóricos. Além disso, foi concluído que quanto maiores forem os valores de pressão arterial, maior será a redução advinda da dieta DASH. Nesse contexto, alguns indivíduos com valores pressóricos $\geq 150 \mathrm{mmHg}$ no componente sistólico obtiveram redução de até $20 \mathrm{mmHg}$ na pressão sistólica (REIS EA, et al., 2021).

Em relação à ingestão de substitutos do sódio na dieta DASH e redução da pressão arterial, muitos estudos epidemiológicos e ensaios clínicos exploraram os efeitos de tal conduta (consumo de sais com baixo teor de sódio e alta concentração de potássio, cálcio e magnésio) na redução da pressão arterial. Uma metanálise mostrou que a utilização dos substitutos do sódio é eficaz tanto na diminuição da pressão sistólica, quanto na diastólica. O resultado disso foi uma redução da ingesta de sódio na população em torno de $33 \%$, bem como uma diminuição de $>10 \mathrm{mmHg}$ na PA sistólica e diastólica, além de uma redução de $75-80 \%$ de AVC e atenuação dos casos de Doença Arterial Coronariana (DAC). Outro estudo analisou a substituição do sódio por cloreto de potássio por dois meses a 3 anos. O resultado foi a diminuição da PAS em $5,7 \mathrm{mmHg}$ e 2,0 $\mathrm{mmHg}$ na PAD, confirmando, assim, o efeito hipotensor do uso de substitutos do sódio. Alguns exemplos de alimentos ricos em potássio são: feijão, laranja, ervilha, leite desnatado, damasco e abacate (HOCK S, et al., 2018; PENG YG, et al., 2014).

\section{CONSIDERAÇÕES FINAIS}

Por meio da análise bibliográfica, verificou-se que a dieta DASH é uma das modificações dietéticas mais largamente prescritas para reduzir a pressão arterial e risco de doenças cardiovasculares, como a DAC e o AVC. Com isso, ela proporciona uma melhor qualidade de vida aos pacientes com HAS, além de gerar redução do peso corporal e da morbimortalidade. Contudo, apresenta algumas dificuldades na sua implementação. Os fatores que contribuem para a adesão incluem a dificuldade de seguir dietas prescritas, a falta de variedade no menu e condições socioeconômicas. Portanto, é importante fornecer opções para a 
dieta DASH que permitam a variação dos nutrientes, mantendo os benefícios sobre a pressão arterial e para saúde em geral.

\section{REFERÊNCIAS}

1. BARROSO WKS, et al. Diretrizes Brasileiras de Hipertensão Arterial - 2020. Arq. Bras. Cardiol. 2021; 116(3): 516658.

2. BRICARELLO LP, et al. Abordagem dietética para controle da hipertensão: reflexões sobre adesão e possíveis impactos para a saúde coletiva. Cien. Saúde Colet., 2020; 25(4): 1421-1432.

3. CHALLA HJ, et al. DASH Diet To Stop Hypertension. StatPearls, 2020.

4. FILIPPOU CD, et al. Dietary Approaches to Stop Hypertension (DASH) Diet and Blood Pressure Reduction in Adults with and without Hypertension: A Systematic Review and Meta-Analysis of Randomized Controlled Trials. Adv. Nutr. Oxford University Press; 2020; 11(5):1150-1160.

5. GEWEHR DM, et al. Adesão ao tratamento farmacológico da hipertensão arterial na Atenção Primária à Saúde. Saúde em Debate. FapUNIFESP; 2018; 42(116):179-190.

6. HOCK S, et al. Tratamento não-farmacológico da hipertensão arterial sistêmica : revisão narrativa. 2018; 2(2): 86-90.

7. JURASCHEK SP, et al. Effects of Sodium Reduction and the DASH Diet in Relation to Baseline Blood Pressure. J. Am. Coll. Cardiol. Elsevier USA; 2017; 70(23): 2841-2848.

8. KUCHARSKA A, et al. The impact of individualised nutritional therapy according to DASH diet on blood pressure, body mass, and selected biochemical parameters in overweight/obese patients with primary arterial hypertension: a prospective randomised study. Kardiol. Pol. Via Medica, 2018; 76(1):158-165.

9. LONGO MAT, et al. Hipertensão arterial sistêmica: aspectos clínicos e análise farmacológica no tratamento dos pacientes de um setor de psicogeriatria do Instituto Bairral de Psiquiatria, no município de Itapira, SP. Rev. Bras. Geriatr. e Gerontol. FapUNIFESP; 2011;14(2):271-284.

10. MACHADO LA, et al. Critérios de escolha de fármacos anti-hipertensivos em adultos. Brazilian Journal of Health Review, 2021; 4(2): 6756-6775.

11. MALACHIAS MVB, et al. $7^{\text {a }}$ Diretriz Brasileira de Hipertensão Arterial: Capítulo 2 - Diagnóstico e Classificação. Arq. Bras. Cardiol. Arquivos Brasileiros de Cardiologia; 2016; 07-13.

12. MALTA DC, et al. Prevalence of and factors associated with self-reported high blood pressure in Brazilian adults. Rev. Saúde Pública. Universidade de São Paulo, 2017; 51:1S-10S.

13. MARIS AS, et al. Interactions of the DASH Diet with the Renin-Angiotensin-Aldosterone System. Curr. Dev. Nutr. Oxford University Press, 2019; 3(9).

14. MELGAÇO NPR, et al. Promoção de Hábitos Saudáveis de Vida para Hipertensos Usando Grupos Operativos, Na Atenção Primária Em Saúde. Brazilian J. Dev. Brazilian Journal of Development, 2021; 7(3): 24538-24548.

15. MOREIRA JPL, et al. Prevalence of corrected arterial hypertension based on the self-reported prevalence estimated by the Brazilian National Health Survey. Cad. Saude Publica. NLM, 2020; 36(1).

16. PASSOS AMV, et al. Hypertension in Brazil: Estimates from Population-Based Prevalence Studies. Epidemiologia e Serviços de Saúde, 2006; 15(1): 35-45.

17. PENG YG et al. Effects of salt substitutes on blood pressure: A meta-analysis of randomized controlled trials. Am. J. Clin. Nutr. American Society for Nutrition, 2014; 100(6): 1448-1454.

18. PIPER AV, et al. Dieta DASH na redução dos níveis de pressão arterial e prevenção do acidente vascular cerebral. Scientia Medica, 2012; 2(22): 113-118.

19. REIS EA et al. Efeitos metabólicos da adoção das abordagens dietéticas para controlar a hipertensão (DASH). Brazilian Journal of Health Review, 2021; 4(4): 14497-14511.

20. SOCIEDADE BRASILEIRA DE CARDIOLOGIA (SBC). $7^{a}$ Diretriz Brasileira de Hipertensão Arterial. Soc. Bras. Cardiol. 2016; 107(3): 103.

21. WESCHENFELDER MD, GUE MJ. Hipertensão arterial: principais fatores de risco modificáveis na estratégia saúde da família Hipertensión arterial: principales factores de riesgo modificables en la estrategia salud de la famila. Enferm. Glob., 2011; 11(26): 354-363. 